\title{
Magnetic reconnection and the lowest energy state
}

\author{
E. N. Parker \\ University of Chicago, Chicago, Illinois, U.S.A. \\ (Received March 14, 2000; Accepted September 29, 2000)
}

\section{Introduction}

Rapid magnetic reconnection occurs when the Maxwell stresses in the magnetic field $\mathbf{B}$ in a highly conducting fluid push the fluid so as to concentrate $\nabla \times \mathbf{B}$ and the associated current density $\mathbf{j}$ into sheets of vanishing thickness and unbounded intensity, i.e. surfaces of tangential discontinuity in B. The essential point is that this condition prevails in the static equilibrium of all but the simplest field topologies because surfaces of tangential discontinuity are an integral part of the lowest available energy state of the field. That is to say, in almost all field topologies static equilibrium, with the Maxwell stresses everywhere in balance, can be achieved only by forming internal surfaces of tangential discontinuity.

The presence of a small resistivity in the fluid prevents the formation of a true discontinuity, of course, so that the magnetic field continually strives to concentrate the associated electric currents, but cannot do so, leaving the field in the dynamical (nonequilibrium) state of rapid dissipation and rapid reconnection across the incipient surface of discontinuity (Parker, 1957, 1994). The dynamical reconnection continues until the topology of the field is reduced to so simple a form that discontinuities are no longer part of static equilibrium. Thus, internal rapid reconnection is intrinsic to the static equilibrium of almost all field topologies, in the laboratory, in the bipolar magnetic fields of the active Sun, the stars, and the Galaxy. That is to say, in the real world, where there is always some slight resistivity, it is not possible for most fields to settle immediately into complete static equilibrium. They must first go through the lengthy process of simplifying their topology through internal reconnection. Since most fields in astronomical settings are subject to continual convective deformation, they are in a perpetual state of internal rapid reconnection and magnetic dissipation, creating such suprathermal phenomena as the solar corona.

The purpose of this lecture is to show this general condition from the magnetic force balance equation $\partial M_{i j} / \partial x_{j}=$ 0 , where $M_{i j}$ is the Maxwell stress tensor $-\delta_{i j} B^{2} / 8 \pi+$ $B_{i} B_{j} / 4 \pi$, representing the isotropic pressure $B^{2} / 8 \pi$ and the tension $B^{2} / 4 \pi$ along the field lines. The more difficult task is the computation of the reconnection (dissipation) rates, presumably starting with the onset of resistive instabilities with the eventual dynamical stagnation flow in two and three dimensions, perhaps in the presence of local plasma turbu-

Copy right(C) The Society of Geomagnetism and Earth, Planetary and Space Sciences (SGEPSS); The Seismological Society of Japan; The Volcanological Society of Japan; The Geodetic Society of Japan; The Japanese Society for Planetary Sciences. lence and anomalous resistivity excited by the concentrated current density $\mathbf{j}$. That we leave to the rest of this conference.

\section{Static Equilibrium}

Consider, then, the formation of surfaces of tangential discontinuity in a magnetic field embedded in an ideal fluid lacking electrical resistivity. To fix ideas consider a uniform magnetic field $B_{0}$ extending from the nonresistive (infinitely conducting) boundary plane $z=0$ through a nonresistive fluid to the nonresistive boundary plane $z=+L$. Holding the footpoints of the field fixed at $z=0$, introduce the two dimensional fluid motion

$$
v_{x}=+k z \partial \psi / \partial y, \quad v_{y}=-k z \partial \psi / \partial x, \quad v_{z}=0,
$$

throughout $L \geq z>0$, where the arbitrary function $\psi=$ $\psi(x, y, k z t)$ is bounded, continuous, with smooth derivatives, etc. The arbitrary motion introduces arbitrary patterns of interlacing of the field lines through the swirling and shuffling of the upper boundary $z=L$. After a time $t$ the magnetic field has the form

$$
\begin{aligned}
& B_{x}=+B_{0} k t \partial \psi / \partial y, \\
& B_{y}=-B_{0} k t \partial \psi / \partial x, \quad B_{z}=B_{0} .
\end{aligned}
$$

Stop the motion at time $t=\tau$. The field is everywhere bounded, continuous, smooth, etc. with arbitrary field line topology, sketched in Fig. 1. Needless to say, the magnetic field is not in static equilibrium.

Then hold both boundary planes $z=0, L$ rigid while the fluid throughout $0<z<L$ is released so that the field may relax to the lowest available energy state. A small viscosity is introduced to provide dissipation, and the fluid pressure is maintained at some uniform value at the boundary planes so that the pressure is uniform throughout the field. Presumably the field relaxes asymptotically as $\exp [-(t-\tau) / T]$ to the lowest available energy state, where $T$ is some finite characteristic time.

In view of the absence of electrical resistivity, the field line topology, i.e. the interlacing of the flux bundles and field lines, is preserved. Thus, clearly, the ultimate equilibrium state of the field exists, has the same topology as the initial field, given by Eq. (1), and the final equilibrium state is unique to that topology. The final state of the field is described by $\partial M_{i j} / \partial x_{j}=0$, or

$$
\nabla \times \mathbf{B}=\alpha \mathbf{B},
$$




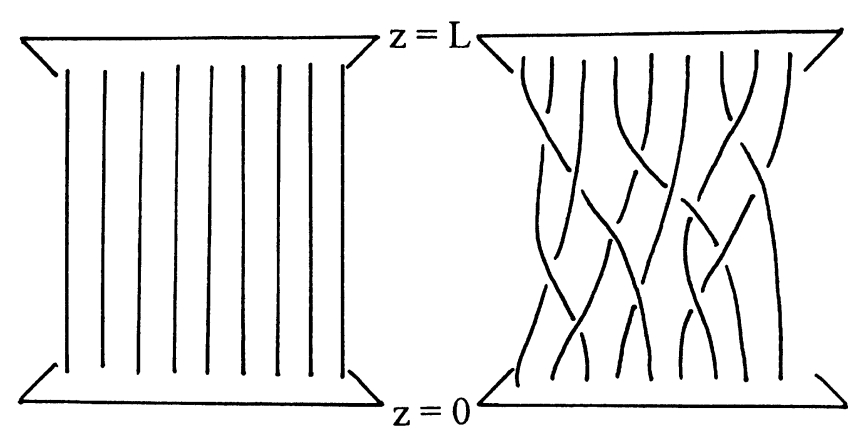

Fig. 1. A sketch of the arbitrary interlace field created by the arbitrary stream function $\psi$ throughout $0<z<L$.

where the "torsion coefficient" $\alpha$ is a scalar function of position.

This familiar force-free equilibrium equation has some remarkable properties, which are the central theme of this lecture. First of all, the equilibrium equation is not linear in spite of its deceptively simple appearance, because $\alpha$ is not independent of $\mathbf{B}$. The divergence of the equilibrium equation yields

$$
\text { B } \nabla \alpha=0
$$

stating that $\alpha$ is constant along each field line and the field lines collectively represent a family of real characteristics of the equilibrium equation (2). The curl of the equilibrium equation yields

$$
\mathbf{B} \times \nabla \alpha=\nabla^{2} \mathbf{B}+\alpha^{2} \mathbf{B} .
$$

The presence of the Laplacian operator indicates two families of complex characteristics, and the equation looks like a second order quasi-linear elliptic equation, except that we know that there is also a family of real characteristics, viz. the field lines (cf. p. 363, Parker, 1979). It is the mixed characteristics that give the equilibrium equation its remarkable properties, quite different from the fully elliptic and fully hyperbolic partial differential equations with which we normally deal. So we must abandon our basic intuition and proceed from here by mathematical "dead reckoning". Thus we find that specification of the field $\mathbf{B}(x, y, 0)$ on the lower boundary determines the field throughout the entire region, including the upper boundary, because the real characteristics extend the solution along the field lines to the upper boundary. In particular, any singularity in the field at the lower boundary propagates along the field lines through the entire region. Conversely, any discontinuity created in the interior extends out to the boundaries $z=0, L$.

To see how this comes about, note that the three components of the equilibrium equation (2) and the divergence condition can be solved for $\partial \mathbf{B} / \partial z$ and $\alpha$ at $z=0$ in terms of the field $\mathbf{B}(x, y, 0)$ at the boundary, yielding

$$
\begin{aligned}
& \frac{\partial B_{x}}{\partial z}=\frac{\partial B_{z}}{\partial x}+\alpha B_{y} \\
& \frac{\partial B_{y}}{\partial z}=\frac{\partial B_{z}}{\partial y}-\alpha B_{x} \\
& \frac{\partial B_{z}}{\partial z}=-\frac{\partial B_{x}}{\partial x}-\frac{\partial B_{y}}{\partial y}
\end{aligned}
$$

$$
\alpha=\frac{1}{B_{z}}\left(\frac{\partial B_{y}}{\partial x}-\frac{\partial B_{x}}{\partial y}\right) .
$$

Differentiating these expressions with respect to $z$ then provides all the higher derivatives with respect to $z$ and the field can be constructed as a series in ascending powers of $z$, given the field $\mathbf{B}(x, y, 0)$ on the lower boundary.

The difficulty is that we do not know the field on the boundary, upper or lower, following the relaxation from the initial form given by Eq. (1) to the final static equilibrium. We know that the field line topology is preserved, and we know that a unique equilibrium state exists. So the essential information for the unique solution is there somewhere, obviously contained in the precise topology of the field. That is the outstanding feature of the equilibrium equation (2).

\section{Optical Analogy}

Given the central role of the field line topology, it is evident that we should understand how a field line propagates (extends) through the region of field. For that we turn to the optical analogy (Parker, 1987a, 1989, 1991, 1994). Draw any curve $C$ across the field, thereby defining a flux surface $S_{c}$, made up of all the field lines intersecting $C$. The flux surface $S_{c}$ represents a two dimension non-Euclidean space, in which the field has no curl. That is to say, if the field in $S_{c}$ had a curl, the curl would be perpendicular to $S_{c}$, whereas the equilibrium equation (2) asserts that there is no component of the curl perpendicular to $S_{c}$. Hence the magnetic field in $S_{c}$ can be written as the gradient of a scalar, $-\nabla \phi$, and the field lines satisfy

$$
B \frac{d X_{i}}{d s}=-\frac{d \phi}{d X_{i}}, \quad(\nabla \phi)^{2}=B^{2}
$$

at each point on $S_{c}$, where $B$ is the magnitude of the field, $X_{i}$ represents a local cartesian coordinate system with origin at the point, and $s$ represents arc length along the field lines. Note then that the eikonal equation for the optical ray path of the wave $\exp i \Phi$ is

$$
n \frac{d x_{i}}{d s}=-\frac{\partial \Phi}{\partial x_{i}}, \quad(\nabla \Phi)^{2}=n^{2}
$$

where $n$ is the index of refraction. It is evident by inspection that the field line has the same path in $S_{c}$ as an optical ray in a medium with index of refraction proportional to $B$. Thus, for instance, the field line is concave toward a local maximum in $B$. Fermat's principle applies and

$$
\delta \int_{1}^{2} d s B=0
$$

for the integral of the "index of refraction" along the path between points 1 and 2. Euler's equation can be written

$$
\frac{y^{\prime \prime}}{\left(1+y^{\prime 2}\right)}=\frac{\partial \ln B}{\partial y}-y^{\prime} \frac{\partial \ln B}{\partial x}
$$

for the simple case of a flat surface, using the rectangular coordinates $(x, y)$ (For surfaces that are not flat, see p. 194, Parker, 1994). 


\section{Local Field Maxima}

The original twisting and interlacing of the field lines, described by the stream function $\psi(x, y, k z t)$, carry over into the final equilibrium state, and the result is an inhomogeneous enhanced magnetic pressure. Each twisted flux bundle expands and crowds its neighbors while the interlacing wraps one flux bundle around another. Denote by $\Delta B$ the variation of the field magnitude from the mean, and note that where one taut flux bundle is drawn around another, sketched in Fig. 2, there is a local maximum in the magnetic pressure and, hence, a local maximum in $\Delta B$. Denote the characteristic width of the maximum by $w$ and the characteristic length by $h$, and consider a field line passing through the region of the maximum between two fixed points at distances $\lambda$ on opposite sides of the maximum $(w, h \ll \lambda)$. The field line follows the "optical" path for which the line integral of $B$ is the least. If the line passes straight across the maximum, the line integral is enhanced by something of the order of $h \Delta B$, whereas, if the line is diverted around the maximum, the path length increases from $2 \lambda$ to $2\left(\lambda^{2}+w^{2}\right)^{1 / 2}$ and the line integral is enhance by $B w^{2} / \lambda$. Thus the line is diverted around the maximum when

$$
\frac{\Delta B}{B}>\frac{w^{2}}{\lambda h}
$$

in order of magnitude.

Diversion of field lines around a local maximum in $\Delta B$ leaves a gap in the flux surface $S_{c}$. The gap extends through a stack of flux surfaces of finite thickness, of course, and the fields on opposite sides bulge through the gap and meet each other somewhere in the middle, sketched in Fig. 3. Since the bulging fields are generally not parallel where they meet, their surface of contact is a surface of tangential discontinuity.

Consider next the effect of twisting a flux bundle. The result is a compression of the central region along the axis of the twisted bundle, as a consequence of the tension in the azimuthal field of the twist. However, the same azimuthal field expands outward and increases the outer radius of the bundle, or, if the bundle is confined to a fixed radius $R$, the total field and the external pressure necessary to confine the bundle are increased (see figure 9.1, p. 172, Parker, 1979).

As an example consider the simple case of a uniform magnetic field $B_{0}$ within the circular boundary $\omega=R$. The field is subjected to the uniform twist, amounting to every line of force making one revolution about the axis of the bundle in a distance $2 \pi a$ along the axis, so that $B_{\varphi} / B_{z}=\omega / a$. Then for force-free equilibrium of the twisted flux bundle the field components are expressible as

$$
B_{z}^{2}(\omega)=f(\omega)+\frac{1}{2} \omega \frac{d f}{d \omega}, \quad B_{\varphi}^{2}(\omega)=-\frac{1}{2} \omega \frac{d f}{d \omega}
$$

in terms of the generating function $f(\omega)$, which represents the square of the field magnitude. Starting with the uniform field $B_{0}$ the longitudinal magnetic flux is $\pi R^{2} B_{0}$. The flux is conserved during the twisting, so that

$$
R^{2} B_{0}=2 \int_{0}^{R} d \omega \omega B_{z}(\omega)
$$

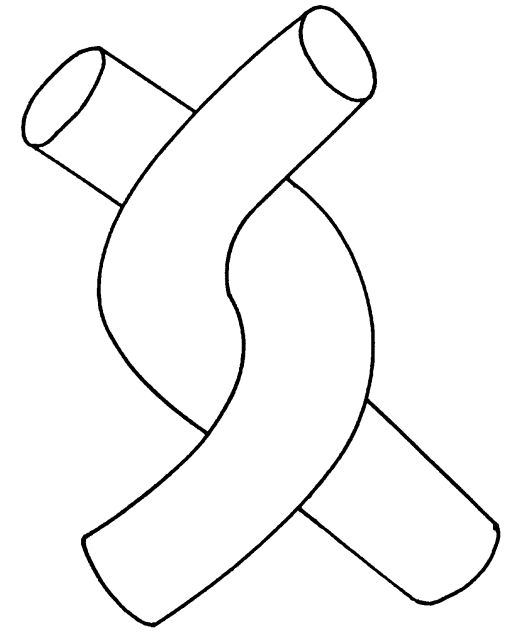

Fig. 2. A sketch of two flux bundles pulled around each other, thereby increasing the magnetic pressure in the vicinity of their contact.

The ratio $B_{\varphi} / B_{z}$ yields

$$
a^{2}\left(-\frac{1}{2} \omega \frac{d f}{d \omega}\right)=\omega^{2}\left(f+\frac{1}{2} \omega \frac{d f}{d \omega}\right)
$$

from which it follows that $f(\omega)=C /\left(a^{2}+\omega^{2}\right)$. The integration constant $C$ has the value $B_{0}\left(R^{4} / a^{4}\right)\left[\ln \left(1+R^{2} / a^{2}\right)\right]^{2}$ so as to conserve total magnetic flux. The magnetic pressure $P=f(R) / 8 \pi$ at the fixed outer boundary $\omega=R$ increases with decreasing $a$ according to

$$
\begin{aligned}
P & =\frac{B_{0}^{2}}{8 \pi}\left(\frac{R}{a}\right)^{4} \frac{1}{\left(1+R^{2} / a^{2}\right)\left[\ln \left(1+R^{2} / a^{2}\right)\right]^{2}} \\
& \approx \frac{B_{0}^{2}}{8 \pi}\left(1+\frac{1}{12} \frac{R^{4}}{a^{4}}+\cdots\right) .
\end{aligned}
$$

So the flux bundles begin to crowd each other, redistributing themselves to give the most efficient close packing, and creating longitudinal ridges of pressure along their sides where they crowd against their neighbors. The essential point is that a ridge of maximum $\Delta B(\ll B)$ refracts field lines so that the inclination $\theta$ of the line to the crest of the ridge as the line crosses the crest is $(2 \Delta B / B)^{1 / 2}$ for grazing incidence $\varepsilon(\ll 1)$ of the line as it approaches the ridge, sketched in Fig. 4. A field line at grazing incidence $-\varepsilon$ from the opposite side of the ridge (in a nearby flux surface) crosses at an inclination $-(2 \Delta B / B)^{1 / 2}$, so that in the limit $\varepsilon \rightarrow 0$ the two lines cross each other at an angle $2(2 \Delta B / B)^{1 / 2}$ at the crest of the ridge. Thus there is a surface of tangential discontinuity of this amount at the flux surface for which $\varepsilon=0$.

\section{Surfaces of Discontinuity}

It is important to understand the nature of the surface of tangential discontinuity because the structure plays an essential role in the physics. The surface of discontinuity is the geometrical surface of contact between the two regions of continuous field on either side. Thus the surface belongs to neither region and so contains no field. It is important to realize, then, that the finite shear in the field across the surface of discontinuity is not reflected in the scalar function $\alpha$, 


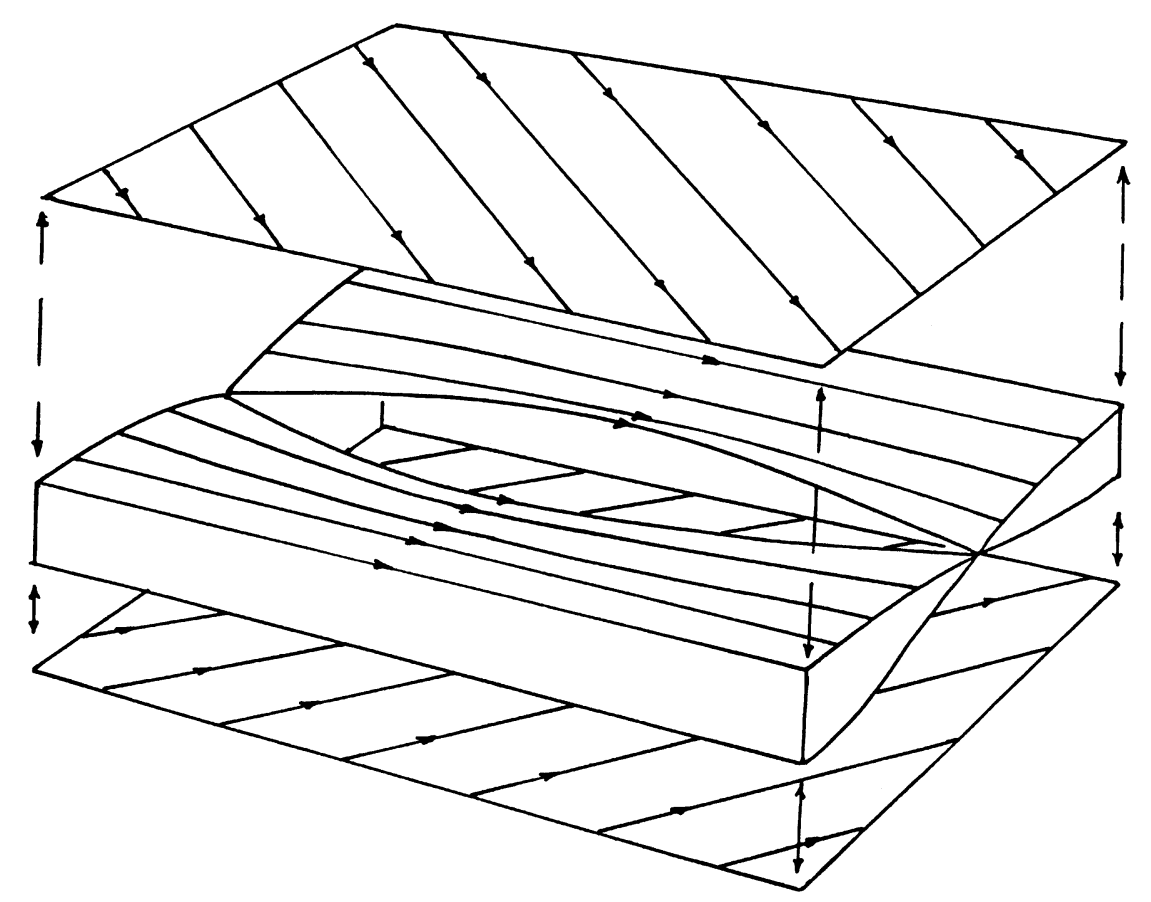

Fig. 3. A sketch of a gap in a layer of flux created by a localized magnetic pressure maximum (see Fig. 2).

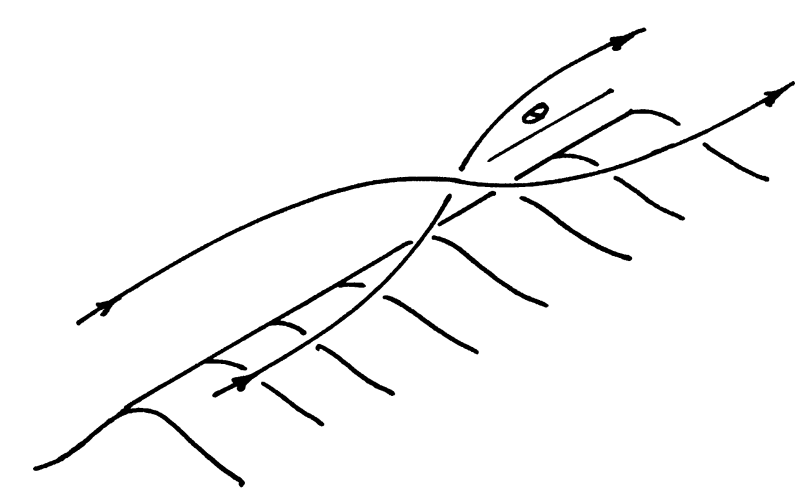

Fig. 4. A sketch of a pressure ridge and the refracted field lines immediately above and below the flux surface in which the field is parallel to the ridge.

which can be defined only in terms of the field $\mathbf{B}$. To appreciate the physical importance of this concept, recall that the initial interlacing of the magnetic field in the fluid motion described by $\psi(x, y, k z t)$ is a purely mechanical manipulation of the fluid and of the field carried in the fluid, allowing the field to be interwoven and interlaced in any arbitrary pattern (opposed only by the limited tension in the field lines). For instance, it is possible to wrap three flux bundles around each other so as to form a braid, in which each flux bundle wraps first one way and then the other around each of the other two. Now in order for the fields of the flux bundles to be continuous where two bundles press against each other, the internal torsion $\alpha$ of each bundle must match the spiraling of the bundle around the contiguous bundle. If the internal torsions of the bundles do not have precisely the correct magnitude and sign to match together where they come in contact, then the contact surface between the two bundles becomes a surface of tangential discontinuity. The problem is that $\alpha$ is required to be constant along each field line $(\mathbf{B} \cdot \nabla \alpha=0)$, so that $\alpha$ cannot vary from one location to the next. It follows that if a bundle spirals first one way and then the other around the flux bundles against which it presses in passing from $z=0$ to $z=L$, the internal torsion cannot be adjusted to fit smoothly to all the other fields. The result is surfaces of tangential discontinuity between the equilibrium flux bundles. Note then that the variable shear across the surfaces of discontinuity does not violate the condition that $\mathbf{B} \cdot \nabla \alpha=0$ because the surface of discontinuity contains no B. Thus if surfaces of discontinuity were not an intrinsic part of the solutions of the equilibrium equation (2), the equilibrium equation would contradict the physical fact that we can braid the flux bundles in any arbitrary way. So the mathematics of the equilibrium equation (2) takes care of the physics, as we know it must.

It is clear from the foregoing optical analogy why the symmetric nonlaced field topologies customarily employed in our analytical calculations do not produce internal surfaces of tangential discontinuity. The field magnitude $B$ simply does not have localized maxima. It is straightforward to produce analytical solutions of Eq. (2) which contain surfaces of discontinuity, either by squeezing a slab of force-free field in some limited neighborhood or by applying a ridge of pressure to a force-free field which somewhere contains a flux surface in which the field lines are parallel to the ridge (Parker, 1987a, 1990 and Chap. 5, Parker 1994).

\section{Conclusion}

Note that all magnetic fields with both ends tied to a slowly convecting body like the Sun are deformed so as to have magnetic free energy and interlaced field lines. The Maxwell stresses in the interlaced field continually strive to produce 
internal surfaces of tangential discontinuity and the slight resistivity of the ambient gas produces dissipation and rapid reconnection at the sites of the incipient surfaces of discontinuity. Thus localized rapid reconnection is a universal phenomenon, common to all convecting stars. Observational studies indicate that this is the origin of flares, microflares, and nanoflares, evidently responsible for solar cosmic rays and for the heating that creates the solar corona and the solar wind and the emission of X-rays, as already noted (cf. Parker, $1987 \mathrm{~b}, 1988,1994)$. Then it should be borne in mind that a slight misalignment within the laboratory magnetic configuration, so that the field topology is not quite as simple as intended for plasma confinement, may provide the onset of weak reconnection, restructuring the field topology in such a way as to provide a growing rapid reconnection situation. So rapid reconnection is an ubiquitous phenomenon, providing for much of the suprathermal phenomena in the astronomical universe and perhaps some of the headaches in the plasma confinement laboratory.

\section{References}

Parker, E. N., Sweet's mechanism for merging magnetic fields in conducting fluids, J. Geophys. Res., 62, 509, 1957.
Parker, E. N., Cosmical Magnetic Fields, 841 pp., Clarendon Press, Oxford, 1979.

Parker, E. N., Magnetic reorientation and the spontaneous formation of tangential discontinuities in deformed magnetic fields, Astrophys. J., 318, 876, 1987a.

Parker, E. N., Stimulated dissipation of magnetic discontinuities and the origin of solarflares, Solar Phys., 111, 297, $1987 \mathrm{~b}$.

Parker, E. N., Nanoflares and the solar X-ray corona, Astrophys. J., 330, 474, 1988.

Parker, E. N., Spontaneous tangential discontinuities and the optical analogy for static magnetic fields. II The optical analogy, Geophys. Astrophys. Fluid Dyn., 45, 169, 1989.

Parker, E. N., Spontaneous tangential discontinuities and the optical analogy for static magnetic fields. III Zones of exclusion, Geophys. Astrophys. Fluid Dyn., 46, 105, 1989.

Parker, E. N., Spontaneous tangential discontinuities and the optical analogy for static magnetic fields. IV High speed fluid sheets, Geophys. Astrophys. Fluid Dyn., 50, 229, 1989.

Parker, E. N., Spontaneous tangential discontinuities and the optical analogy for static magnetic fields. V Formal integration of the force-free field equations, Geophys. Astrophys. Fluid Dyn., 52, 183, 1990.

Parker, E. N., The optical analogy for vector fields, Phys. Fluids B, 3, 2652, 1991.

Parker, E. N., Spontaneous Current Sheets in Magnetic Fields, 420 pp., Oxford University Press, New York, 1994.

E. N. Parker (e-mail: parker@odysseus.uchicago.edu) 